\title{
Mutant TP53 disrupts age-related accumulation patterns of somatic mutations in multiple cancer types
}

\author{
Wensheng Zhang ${ }^{1}$, Erik K. Flemington ${ }^{2}$, Kun Zhang ${ }^{1 \S}$ \\ ${ }^{1}$ Department of Computer Science, Xavier University of Louisiana, New Orleans, LA, USA \\ ${ }^{2}$ Tulane Cancer Center, Tulane University, New Orleans, LA, USA \\ $\S$ Corresponding author
}

\begin{abstract}
Most cancers are driven by somatic mutations in proto-oncogenes and tumor suppressor genes. Genetic changes in a tumor may accumulate in the tissue self-renewal phase prior to neoplasm. The risk of sporadic mutations increases with age. In this regard, a positive association between patient age and the accumulated mutation burden in tumors exists for many cancer types. However, the reported evidences for such a connection are still limited. TP53 is the most frequently mutated cancer gene. The encoded p53 protein plays crucial roles in DNA repair. Hereby, we speculate that mutant TP53 can disrupt the age-related accumulation patterns of somatic mutations in tumors. We performed linear model analyses on the clinically-annotated genomic data published by TCGA. We found that there was a significant interaction between TP53 genotype (mutant versus wild-type) and patient age at the initial clinical date on somatic mutation burden for five cancers. That is, the regression coefficients of mutation burden on patient age were significant $(p<0.05)$ for TP53 wild-type tumors but not the mutant counterparts. This disparity was further verified by comparing the group-specific regression coefficients. This finding confirmed our hypothesis and provided unique insights into p53-related tumorigenesis such as the potential temporal order of driver mutations.
\end{abstract}

\section{Introduction}

The incidence of most forms of adult-onset cancers increases dramatically as a function of age in mammals $(1,2)$. More than half of them are detected in persons aged 65 years or older $(3,4)$. In light of the classical multi-mutation theory of tumorigenesis $(2,5)$, which states that cancer is the result of accumulated mutations in a cell's DNA, the age-related pattern of cancer incidences can be explained by several mutually complementary mechanisms. First, sporadic mutation risk increases with age (6-8). Second, some fraction of the genetic changes present in a tumor is accumulated in the tissue selfrenewal phase prior to neoplastic process (9). In this regard, a positive association between patient age and the number of accumulated mutations in tumors could exist across a wide spectrum of cancer types. Such a relationship has been suggested by recent publications (9-11), while the reported evidences have yet been limited. That is, the demonstrated correlation was significant in only a fraction of the addressed cancer types and the findings warrant further validation using additional data and/or alternative statistical methods. Hence, the universal applicability of a model relating mutation accumulation to patient age should be toned down as there are exceptions, e.g. no age-mutation association was found in chronic myelomonocytic leukemia (CMML) for which $<5 \%$ of patients have a TP53 mutation (12) although such a relationship was reported in acute myeloid leukemia (AML) (11). On the other hand, the statistical analysis results could be further refined by introducing other major biological or genetic factors into the model.

(C) 2016. This manuscript version is made available under the Elsevier user license

http://www.elsevier.com/open-access/userlicense/1.0/ 
The tumor suppressor TP53 (p53) is the most frequently altered gene in human cancers (13). The loss or disruption of p53 function due to a mutation can lead to uncontrolled cell proliferation and eventually cancer (14). Considering the crucial roles of normal p53 in conserving genome stability, we speculate that its aberration can disrupt or reshape age-related accumulation patterns of somatic mutations in tumors. To test this hypothesis, we performed a set of linear model analyses on the genomic and clinical data for multiple cancer types released by the Cancer Genome Atlas (TCGA, http://cancergenome.nih.gov).

\section{Material and Methods}

Among the 33 cancer types with clinically-annotated multi-omic data (available at TCGA database by April 24, 2015), twelve were studied in this work by considering the genetic diversity of patients and the prevalence of somatic TP53 mutations in these tumors. Each of the selected cancer types had at least 15 patients from a minority population (i.e. black American or Asian) besides the dominant white Americans, and the ratio of samples with TP53 non-synonymous mutations was over $25 \%$. The studied cancer types included bladder urothelial carcinoma (BLCA), glioblastoma multiforme (GBM), head and neck squamous cell carcinoma (HNSC), lung adenocarcinoma (LUAD), lung squamous cell carcinoma (LUSC), breast invasive carcinoma (BRCA), ovarian serous cystadenocarcinoma (OV), uterine corpus endometrial carcinoma (UCEC), colon adenocarcinoma (COAD), esophageal carcinoma (ESCA), liver hepatocellular carcinoma (LIHC), and stomach adenocarcinoma (STAD). The sample sizes of those cancer types ranged from 171 to 967 (see Supplemental Table $1)$.

The (somatic) mutation calls (level-2 data) from the TCGA pipeline were directly utilized. For each cancer type, TCGA collected multiple somatic mutation datasets (versions). Those data, contributed by different institutes, were generated using various sequencing platforms, somatic mutation calling algorithms and computational tools. Except for the ovarian carcinomas (OV), we chose one representative dataset for each cancer based on a set of selection criteria (see Supplemental Text 1). For OV, we employed the datasets from Massachusetts Institute of Technology and Washington University in St. Louis. The basic information of the used somatic and clinical datasets was summarized in Supplemental Table 2. Synonymous mutations and those under the categories of "intron" and "rna" were excluded from further analysis. Moreover, we removed the top and bottom $1 \%$ of patient data by their total number of somatic mutations, with an additional filter of remaining patients having more than $1024\left(2^{10}\right)$ mutations. Such hypermutated cases accounted for $3 \sim 13 \%$ of patients in several cancer types including BLCA, LUAD, UCEC and COAD.

A linear regression model with heterogeneous slopes was used to evaluate the effect of patient age (at the initial clinical date) and TP53 genotype of tumor cells on the mutation burden, as well as the interaction between these two explanatory variables. For a tumor, the TP53 genotypes were exclusively determined by the somatic mutation data. In the modeling, mutation burdens (y) were quantified by the log2-transformed total number of non-synonymous somatic mutations. The vector of patient ages was centered by subtracting the mean $(\bar{x})$. The TP53 status (i.e. wild-type versus mutant) of a tumor (patient) was indexed by $i(i=1,2)$.

$$
y_{i j}=\mu+I_{j} \alpha+\beta_{i}\left(x_{j}-\bar{x}\right)+e_{i j} \quad e_{i j} \sim N\left(0, \sigma_{e}\right)
$$

In equation (1), $y_{i j}$ and $x_{j}$ represented the status-specific mutation burden on the $j^{\text {th }}$ tumor sample and the age of the $j^{t h}$ patient, respectively. $I$ was a binary variable with a value of 1 or 0 , indicating the mutant or wild-type TP53 tumors. The 
baseline was denoted by $\mu$ and could be considered as the expected mutation burden for a TP53 wild-type tumor carried by a patient aged at $\bar{x}$. a represented the effect of TP53 mutation on $y$, i.e. the age-adjusted average increase or decrease (when the value was negative) in mutation burden for a tumor with mutated TP53. $\mu+\alpha$ stood for the expected mutation burden for a mutant TP53 tumor carried by a patient aged at $\bar{x}$. The regression coefficient, $\beta_{i}(\mathrm{i}=1,2)$, denoted the increase or decrease (when the value was negative) in mutation burden due to a one-year increase in patient age for wild-type TP53 tumors (when $\mathrm{i}=1$ ) or for mutant-TP53 tumors (when $\mathrm{i}=2$ ). The $\mathrm{p}$-values we were primarily interested in were calculated by four t-tests with $H_{0}: \alpha=0, H_{0}: \beta_{1}=0, H_{0}: \beta_{2}=0$ and $H_{0}: \beta_{2}-\beta_{1}=0$ being the null hypothesis, respectively. We performed the statistical computation using the statistical function $\operatorname{lm}()$ included in the R package "stat". The conventional significance threshold was set to be $p<0.05$.

\section{Results}

The results of the proposed linear model analysis are summarized in Table 1 in the manner of local linear approximation and are visualized in Figure 1. The exact results, including the parameter estimates and data profiles, are presented in Supplemental Table 3 and Supplemental Figure 1. Based on the estimates of model parameters and their p-values, we partitioned the twelve cancer types into four groups, as indicated by the color bars in the first column of Table 1 and Supplemental Table 3. The first group included LIHC, OV, STAD, BLCA and UCEC, in which the regression coefficients (slopes) of mutation burden on patient age were positive and significant only for the TP53 wild-type tumors. These disparities were further confirmed by the statistical tests of the differences of the paired coefficients. Except for UCEC, $\beta_{2}-\beta_{1}$ was significant in the other four cancers. Similarly, besides STAD, TP53 genotype effect ( $a$ in Supplemental Table 3) was significant in four cancers of this group. The second group only contained one cancer, i.e. LUAD, in which the slope was negative and significant in the mutant TP53 tumors but not in the wild-type counterparts. The third group, consisting of BRCA, GBM and HNSC, demonstrated consistent positive association between mutation burden and patient age. The paired regression lines were almost parallel in these cancers. The remaining three cancers, i.e. COAD, ESCA and LUSC, were considered as the fourth group, as they hardly showed any significant patterns regarding the relationship between mutation burden and patient age. It is worth noting that the small p-value (0.011) of $\beta_{2}$ in ESCA was not a strong evidence for a significant regression of mutation burden on age because of the lack of compatibility with the estimates of other parameters.

We noticed that both UCEC and COAD were also studied in (9), where an older version of TCGA somatic mutation spectra was used and COAD was termed as colorectal cancer. As shown in Figures $2 B$ and $2 C$ of the publication, for UCEC (or COAD), tumors with more than 200 (or 250) somatic mutations were not included in the analysis. Using the Kendall's correlation method, Tomasetti et al (2013) found a significant positive correlation $(\mathrm{p}<0.001)$ between the number of somatic mutations and patient age. Here, we repeated our linear model analysis on an "alternative" dataset similar to that used in (9), in which the tumors with more than 200 (or 250) mutations in UCEC (or COAD) were also eliminated. As shown in Supplemental Figure 2, this reanalysis confirmed our results that the TP53 genotype-dependent positive association between mutation burden and patient age did exist in UCEC. For COAD, the positive correlation between mutation burden and patient age reported by (9) was observed in the mutant TP53 tumors, with the p-value being smaller and significant (i.e. 0.002 in Supplemental Figure 2 versus 0.085 in Table 1). Meanwhile, such an association was not present in the wild-type TP53 tumor patients $(\mathrm{p}=0.53)$. 


\section{Discussion}

As mentioned above, of the twelve cancers studied here, eight demonstrated significant age-related accumulation patterns of somatic mutations. This result provided new evidence for the classical multi-mutation theory of tumorigenesis. More importantly, the fact that most of these patterns only appeared in TP53 wild-type tumors confirmed our hypothesis about the interference of TP53 alteration to the mutation accumulation process. Considering that mutant-TP53 tumors had a heavier mutation burden than TP53 wild-type tumors in ten cancers ( $\alpha$ in Supplemental Table 3), it is plausible to predict a burst of genetic alterations in cells following the disruption of function of TP53 gene. If this is true, the time when the TP53 mutations occur in cancerous cells may be a crucial factor to the variability of age-related accumulation patterns of somatic mutations over different types of cancer. Here, we give a speculative explanation. For simplicity but without loss of generality, we assume a scenario where tumorigenesis is driven by three driver-mutations (or driver clusters) DM-a, DM-b and DM-c that arise and confer new fitness advantage to the recipient cells (15) in the early, intermediate or late phase, respectively. In the case that the altered TP53 is DM-a, many genetic changes from the subsequent mutation burst will be fixed as hitchhikers in forming new clone(s) that get additional fitness advantage from DM-b. Meanwhile, the accumulation of moderately deleterious passenger mutations $(16,17)$ may restrict the future increase of mutation burden of a single cell. As a result, the age-related accumulation patterns of somatic mutations are likely hidden from statistical detection. This is what we observed in LIHC, OV, STAD and BLCA. On the other hand, when the altered TP53 is DM-b or DM-c, the fixation of genetic changes arising from the mutation burst will happen during a time period close to the initial clinical date (or the tumor tissue sampling date). In this context, the TP53 genotype will not impact the regression of mutation burden on patient age, which is the case in BRCA, GBM and HNSC.

In COAD, ESCA and LUSC, the TP53 wild-type tumors didn't demonstrate the expected age-related accumulation patterns for somatic mutations, suggesting the existence of other "mutators" that, similar to TP53 mutation, could impact the stability of other genes (18). The patterns in UCEC and LUAD are still hard to explain. In the former, the TP53 wild-type tumors of patients 50 to 70 years old could be clearly divided into a high or low mutation subgroup (see Supplemental Figure 1). In the latter, mutation burden was negatively associated with patient age for the mutant-TP53 tumors while it was insignificant for the wild-type counterparts.

\section{Conclusion}

In this study, we found that mutant TP53 could disrupt or reshape age-related accumulation patterns of somatic mutations present in tumors. We also observed that mutant-TP53 tumors had a heavier mutation burden than TP53 wild-type tumors in ten cancer types, implicating the existence of a burst of genetic alterations in cells after the disruption of function of TP53 gene. As such, we proposed that the time when the TP53 mutations occur in cancerous cells may be a determining factor to the variability of age-related accumulation patterns of somatic mutations over different cancers. We further showed that this hypothesis has a basis in the recently emerged cancer evolution theories. These findings and reasoning provided unique insights into p53-related tumorigenesis.

\section{Acknowledgements}

This publication was made possible by funding from the NIH NIMHD-RCMI grant \#5G12MD007595, the DOD ARO grant \# W911NF-15-1-0510 and the Louisiana Cancer Research Consortium (LCRC). EKF was supported by the NIH 
grants 5R01AI101046 and 5R01AI106676. The analyses presented here are based upon the data published by The Cancer Genome Atlas (TCGA) managed by the NCI and NHGRI. Information regarding TCGA can be found at http://cancergenome.nih.gov. We are also grateful to the reviewers for their constructive comments. We are grateful to the anonymous reviewers for their valuable comments that greatly improved this paper.

\section{Author contributions}

Conceived and designed the experiments: WZ, KZ. Performed the experiments: WZ. Analyzed the data: WZ, KZ. Wrote the paper: WZ, EKF, KZ. All authors read and approved the final manuscript.

\section{Reference}

1. Bunz F (2008) Principles of cancer genetics (Springer, Dordrecht) pp xi, 325 p.

2. Nordling CO. A new theory on cancer-inducing mechanism. Br J Cancer 1953; 7(1):68-72.

3. Anisimov VN. The relationship between aging and carcinogenesis: a critical appraisal. Crit Rev Oncol Hematol 2003; 45(3):277-304.

4. $\quad$ Denduluri N \& Ershler WB. Aging biology and cancer. Semin Oncol 2004; 31(2):137-148.

5. Knudson AG, Jr. Mutation and cancer: statistical study of retinoblastoma. PNAS 1971; 68(4):820-823.

6. Kennedy SR, Loeb LA, \& Herr AJ. Somatic mutations in aging, cancer and neurodegeneration. Mech Ageing Dev 2012; 133(4):118-126.

7. Curry J, Karnaoukhova L, Guenette GC, \& Glickman BW. Influence of sex, smoking and age on human hprt mutation frequencies and spectra. Genetics 1999; 152(3):1065-1077.

8. Martin GM, et al. Somatic mutations are frequent and increase with age in human kidney epithelial cells. Hum Mol Genet 1996; 5(2):215-221.

9. Tomasetti C, Vogelstein B, \& Parmigiani G. Half or more of the somatic mutations in cancers of self-renewing tissues originate prior to tumor initiation. PNAS 2013; 110(6):1999-2004.

10. Tan H, Bao J, \& Zhou X. Genome-wide mutational spectra analysis reveals significant cancer-specific heterogeneity. Sci Rep 2015; 5:12566.

11. Welch JS, et al. The origin and evolution of mutations in acute myeloid leukemia. Cell 2012; 150(2):264-278.

12. Mason CC, et al. (2016) Age-related mutations and chronic myelomonocytic leukemia. Leukemia 30(4):906-913.

13. Vogelstein B, Sur, S. \& Prives, C. p53 : The Most Frequently Altered Gene in Human Cancers. Nature Education 2010;6(3).

14. George P. p53 how crucial is its role in cancer. Int J Curr Pharm Res 2011; 3(2):19-25.

15. Bozic $\mathrm{l}$, et al. Accumulation of driver and passenger mutations during tumor progression. PNAS 2010; 107(43):18545-18550.

16. McFarland CD, Korolev KS, Kryukov GV, Sunyaev SR, \& Mirny LA. Impact of deleterious passenger mutations on cancer progression. PNAS 2013; 110(8):2910-2915.

17. Zhang W, Edwards A, Flemington E, \& Zhang K. Somatic mutations favorable to patient survival are predominant in ovarian carcinomas. PLoS One 2014; 9(11):e112561.

18. Youn A \& Simon R. Using passenger mutations to estimate the timing of driver mutations and identify mutator alterations. BMC Bioinformatics 2013; 14:363.

\section{Caption of Figures}

Figure 1. Illustration of heterogeneous and homogenous regression coefficients (slopes) of mutation burdens on the ages of patients at the initial clinical dates over twelve cancer types. Mutation burdens are measured by the $\log -2$ transformed numbers of somatic mutations that are labeled on the right side of each plot. The red squares (or black circles) represent tumors with (or without) TP53 mutations. The red and black lines represent the regression lines for the two genotypes, respectively. The age domain was divided into several bins (e.g. 15-24, 
25-34, 35-44 ...) and the patients within the same bin are aggregated to be a "macro sample" with the mutation burden being calculated as the average of the contained tumors. Average mutation burden of patients by decile age group (e.g. 15-24, 25-34, 35-44, ...) are plotted with regression lines obtained from fitting the nonaggregated data for the two genotype groups, respectively. Please refer to Supplemental Figure 1 for the illustration of the non-aggregated data. 
LIHC

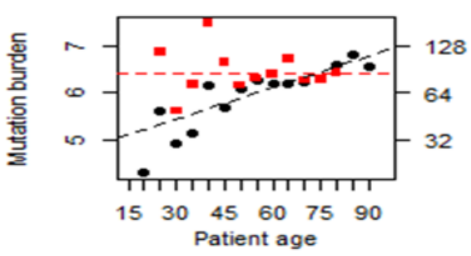

BLCA

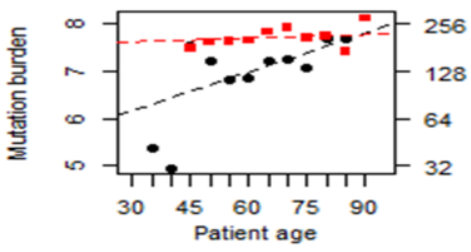

BRCA

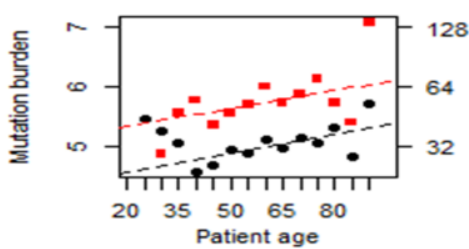

COAD

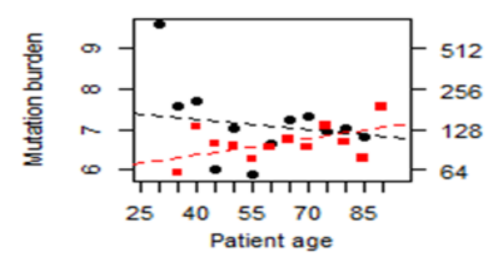

OV

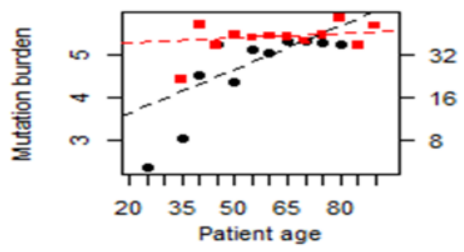

UCEC

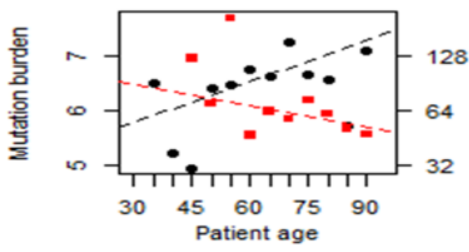

GBM

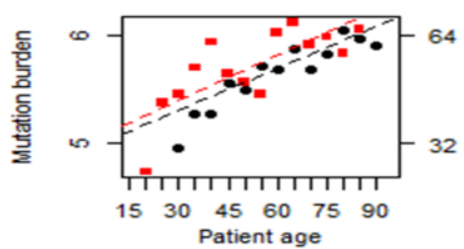

ESCA

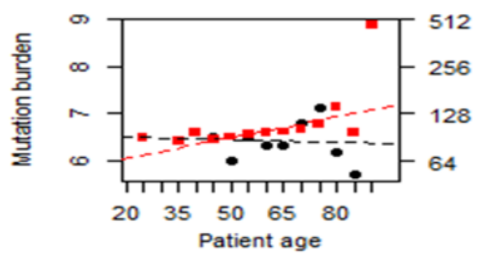

STAD
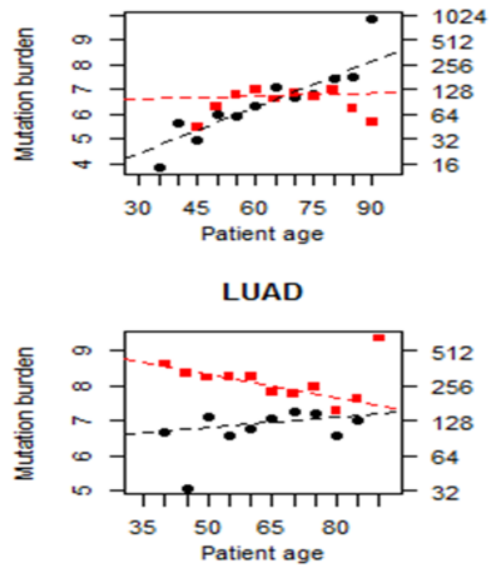

HNSC

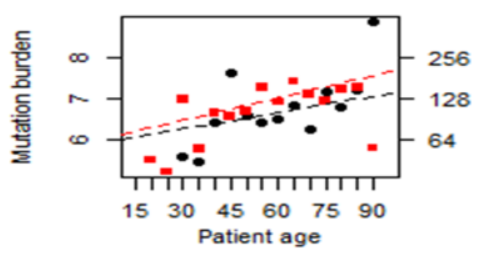

LUSC

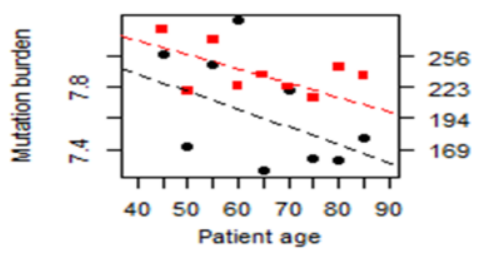


Table 1. Summary of the effects of TP53 genotypes on mutation burden and the age-related accumulation of mutations

\begin{tabular}{|c|c|c|c|c|c|c|c|c|c|}
\hline Cancers & Avg-age & Mut-wd & Mut-mut & P-1 & S-wd & P-2 & S-mut & P-3 & $\mathrm{P}-4$ \\
\hline LIHC & 61 & 69 & 85 & $3.9 \times 10^{-2}$ & 1.1 & $2.2 \times 10^{-5}$ & 0.0 & $9.7 \times 10^{-1}$ & $4.8 \times 10^{-2}$ \\
\hline OV & 59 & 31 & 43 & $2.0 \times 10^{-4}$ & 0.7 & $6.8 \times 10^{-5}$ & 0.1 & $4.3 \times 10^{-1}$ & $1.5 \times 10^{-3}$ \\
\hline STAD & 66 & 101 & 107 & $6.6 \times 10^{-1}$ & 4.4 & $5.6 \times 10^{-8}$ & 0.3 & $7.5 \times 10^{-1}$ & $9.4 \times 10^{-4}$ \\
\hline BLCA & 68 & 145 & 213 & $2.0 \times 10^{-5}$ & 2.8 & $3.0 \times 10^{-4}$ & 0.4 & $7.7 \times 10^{-1}$ & $3.5 \times 10^{-2}$ \\
\hline UCEC & 64 & 97 & 66 & $1.3 \times 10^{-2}$ & 1.7 & $1.6 \times 10^{-2}$ & -0.6 & $4.7 \times 10^{-1}$ & $6.7 \times 10^{-2}$ \\
\hline LUAD & 66 & 127 & 254 & $2.2 \times 10^{-15}$ & 0.8 & $3.0 \times 10^{-1}$ & -4.0 & $7.0 \times 10^{-3}$ & $9.9 \times 10^{-3}$ \\
\hline BRCA & 58 & 31 & 53 & $5.4 \times 10^{-22}$ & 0.2 & $6.0 \times 10^{-4}$ & 0.4 & $5.1 \times 10^{-2}$ & $9.0 \times 10^{-1}$ \\
\hline GBM & 61 & 52 & 57 & $4.8 \times 10^{-2}$ & 0.5 & $1.3 \times 10^{-5}$ & 0.5 & $8.7 \times 10^{-5}$ & $8.8 \times 10^{-1}$ \\
\hline HNSC & 61 & 101 & 129 & $3.8 \times 10^{-4}$ & 0.9 & $6.3 \times 10^{-2}$ & 1.6 & $4.6 \times 10^{-5}$ & $6.0 \times 10^{-1}$ \\
\hline COAD & 65 & 132 & 104 & $3.2 \times 10^{-2}$ & -0.8 & $3.7 \times 10^{-1}$ & 1.0 & $8.5 \times 10^{-2}$ & $7.3 \times 10^{-2}$ \\
\hline ESCA & 62 & 86 & 102 & $5.7 \times 10^{-2}$ & -0.1 & $8.4 \times 10^{-1}$ & 1.0 & $1.1 \times 10^{-2}$ & $1.2 \times 10^{-1}$ \\
\hline LUSC & 68 & 190 & 231 & $3.5 \times 10^{-2}$ & -1.5 & $3.7 \times 10^{-1}$ & -1.5 & $2.2 \times 10^{-1}$ & $8.8 \times 10^{-1}$ \\
\hline
\end{tabular}

*Avg-age: the average patient age at the initial clinical date. Mut-wd: the average mutation number of patients aged Aveage without somatic TP53 mutations. Mut-mut: the average mutation number of patients aged Avg-age with somatic TP53 mutations. P-1: the p-value for the difference of mutation burden between the wild-type and mutant TP53 tumors. S-wd: the local linear approximation of the yearly increase or decrease of mutation number for the wild-type TP53 patients whose ages are close to the average of the entire cohort. P-2: the p-value for the regression coefficient of mutation burden on patient age in wild-type TP53 tumors. S-mut: the local linear approximation of the yearly increase or decrease of mutation number for the mutant TP53 patients whose ages are close to the average of the entire cohort. P-3: the p-value for the regression coefficient of mutation burden on patient age in the wild-type TP53 tumors. p-4: the p-value for the difference of the regression coefficients of mutation burden on patient age between the wild-type TP53 and mutant TP53 sub-groups. Pvalues $<0.05$ : bold; P-values $<0.001$ : bold and red; $0.05<\mathrm{P}$-values $<0.1$ : italic. 